\title{
Das Problem \\ der transzendetalen Zeitbestimmung im Rahmen von Kants Philosophie
}

\section{El problema de la determinación trascendental temporal en el marco de la filosofía kantiana}

\author{
ALBA JIMÉNEZ RODRÍGUEZ \\ Universidad Autónoma de Madrid
}

Recibido: 7-XII-2013 Aceptado: 6-VI-2014

\section{RESUMEN}

El presente artículo constituye un estudio sobre el concepto de determinación trascendental temporal expuesto por Kant en el marco del esquematismo trascendental kantiano en KrV. Con este objeto, se exponen las razones de la preminencia de la definición de esquema como transzendentale Zeitbestimmung, frente a otras asociadas a la interpretación estática del esquematismo y, fundamentalmente, frente aquella donde se presenta como un proceso de subsunción o de aplicación de las categorías a las intuiciones puras, reconstruyendo a su vez el alcance de dicha noción a partir del análisis del estatuto de la determinación temporal en otras obras del corpus kantiano.

\section{PALABRAS CLAVE}

DETERMINACIÓN TRASCENDENTAL TEMPORAL, ESQUEMA TRASCENDENTAL, APLICACIÓN, KANT, HEIDEGGER.

\begin{abstract}
In this paper, I focus on the concept of transcendental time-determination presented by Kant in relation with the transcendental schematism in $\mathrm{KrV}$. With this aim, I discuss why the definition of «schema» as transzendentale Zeitbestimmung is more relevant than other kinds of definitions
\end{abstract}

(C) Contrastes. Revista Internacional de Filosofía, vol. XX-Nº1 (2015), pp. 83-101. ISSN: 1136-4076

Departamento de Filosofía, Universidad de Málaga, Facultad de Filosofía y Letras Campus de Teatinos, E-29071 Málaga (España) 
i.e., the one derived from a static interpretation of schematism and the one where schematism is understood as a process of subsumption or application of categories to pure intuitions. Finally, I reconstruct the role of the schema through an analysis of time-determination as it is developed in the rest of Kant's corpus.

KEYWORDS

TRANSCENDENTAL TIME-DETERMINATION, TRANSCENDENTAL SCHEMA, APPLICATION, KANT, HEIDEGGER.

Von ALlen Bedeutungen, unter denen der Begriff des transzendentalen Schemas in der KrV auftritt, hat anscheinend die Definition als transzendentale Zeitbestimmung Vorrang vor den übrigen, was auf die unauflösliche Verbindung zwischen Zeitlichkeit und dem Problem der Anwendung der reinen Begriffe auf die Anschauungen hindeutet, die die transzendentale Deduktion dem Schematismuskapitel vorwegnimmt. ${ }^{1}$

Des Weiteren soll die Definition des Schemas als transzendentale Zeitbestimmung den Ausgangpunkt darstellen, von dem aus die übrigen Bedeutungen dieses Terminus ausgelegt werden sollen. Da die Schemata nicht nur Bilder für Begriffe aller Art liefern, sondern auch die Bedingung für jegliche Vereinigungsform der Pluralität als synthetische Einheit der anschaulichen Mannigfaltigkeit dank der Wirkung der Zeit, verstanden als die allgemeinste Form der Apprehension (sogar über dem dem äußeren Sinn zugeordneten Raum), kann geschlussfolgert werden, dass die transzendentale Zeitbestimmung die Grundlage für die übrigen, die Prozesse der Anwendung und der synthetischen Gesetzgebung bestimmenden Formen zur Definition des Schemas darstellt.

In jeder dieser transzendentale Bestimmungen wird die Zeit als reines Bild oder Anblick definiert (weshalb Kant die Aufgabe der Einbildungskraft als Exhibition eines Begriffs als subiecto sub aspectum in KU bezeichnet) ${ }^{2}$, wobei dieses Bild eine Instanz darstellt, die aufgrund zahlreicher Elemente den «Eĩ $\delta o \zeta »$ beinhaltet oder beinhalten kann, der alle Elemente nach einer Konstruktionsregel wieder zusammenbringt. Bei dieser Regel handelt es sich um eine Methode zur Bildung einer Pluralität über eine figürliche Synthese.

Die Frage nach einer möglichen Verbindung zwischen dem Logischen (und somit Notwendigen, Universellen und Unveränderlichen) und dem Zeitlichen (und folglich dem Kontingenten und Veränderlichen) bzw. die Frage, ob Wahrheit und Geschichte vielmehr unabänderlich gespalten und der Grund ihrer beiderseitigen Beteiligung vollkommen unerkennbar erscheinen soll, ist ein Thema, das unserer Ansicht nach im Kant'schen Kritizismus und insbesondere

$1 C f: \mathrm{KrV}(\mathrm{A} 137 / \mathrm{B} 176-\mathrm{A} 147 / \mathrm{B} 187)$

2 Cf:: Ak, VIII, 267, (BXLIX- AXLVII). 
in der Lehre vom transzendentalen Schematismus, wo der Begriff der transzendentalen Zeitbestimmung entwickelt wird, auf besondere Art und Weise zum Ausdruck kommt. In der Aufgabe der Kritik selbst wird dieses Spannungsfeld als eine kontinuierliche Übergangsbewegung beibehalten, die nicht als ein bloßes äußeres, statisches und nach der angesprochenen ursprünglichen Trennung aufgekommenes tertium quid dargestellt werden kann, sondern die dieses Spannungsfeld als konstante dogmatische Projektion und skeptische Rückkehr aufrechterhalten muss. Wenn nun diese reflektierende und asymptotische Vermittlung, wie von Kant gewollt, als Geschichte der reinen Vernunft Gestalt annimmt, ist dies eben darauf zurückzuführen, dass die Vernunft a priori den Sinn und die Orientierung ihrer eigenen Abfolge bestimmt und gleichzeitig als das Ergebnis dieses Flusses der Zeit angesehen werden kann, da nämlich das transzendentale Schema, als transzendentale Zeitbestimmung, ein notwendiges Universalitätsmoment mit sich führt, gleichzeitig aber synthetisch unter zeitlichen Bedingungen angewandt wird. Dieses Schema stellt somit den Horizont oder das einheitliche und antizipierbare Ganze dar, von dem ausgehend die Konstruktionsweise dieses Vernunftsystems zu entschlüsseln ist.

Somit kann das System der gesamten Kant'schen Lehre (wenn es denn als geschlossene und selbstbezügliche Erkenntnis auch außerhalb des Grundsatzes der Postulate des empirischen Denkens überhaupt bestünde, in denen diese von der Vernunft antizipierte kollektive Einheit zum Behuf der Erfahrung abgebildet wird) architektonisch nach der in der Schematismuslehre enthaltenen Idee angeordnet werden.

So steht das Problem der transzendentalen Zeitbestimmung, das im Schematismuskapitel vorgestellt wird, im engen Zusammenhang nicht nur mit der Aufstellung der transzendentalen Deduktion der Kategorien und Grundsätze, sondern auch mit der Anerkennung der zwischen dieser Lehre und scheinbar ungleichen Teilen des Kantschen Systems, wie dem Begriff reflektierende Urteile in der $\mathrm{KU}^{3}$, der dritten Antinomie von $\mathrm{KrV}$, wo der Begriff der Zurech-

3 Das transzendentale Schema ist in der Tat auch ein Beispiel für die richtige Anwendung der Urteilskraft. Diese Definition ist in der Rechtssprache ausgedrückt, der Kant üblicherweise seine Metapher entnahm. Zur Anwendung eines Straftatbestands müssen zuvor bestimmte Voraussetzungen der Rechtlichkeit, des Handlungsausschlusses, der Unzurechnungsfähigkeit, usw. bekannt sein. Das heißt, die Begriffe benötigen reflexive Vermittlungsverfahren sowohl für ihre Bildung als auch für ihre mögliche Bezugnahme und Anwendung auf die Erfahrung. Das Urteil wird erneut zu einem Vermittlungselement, zu einem Verbindungsmittel zwischen dem theoretischen und dem praktischen Gebrauch der Vernunft. Die Urteilskraft ist wiederum die Vermittlung der Vermittlung, da sie den Verweis des Begriffes auf die Anschauung, bei der der Begriff schon mittelbar mit dem Objekt in Verbindung tritt, bekräftigt. Der Begriff als eine durch den Satz vom Widerspruch begrenzte Zuweisung, und somit als Denkvorgang, bestimmt die in Form verschiedener Repräsentationen gegebene Funktion der Einheit. Es ist jedoch das Urteil, 
nung auf tritt, der Analytik des Erhabenen ${ }^{4}$, dem Begriff der Konstruktion der Mathematik in den vorkritischen Schriften Kants, dem Begriff des Wärmestoffs im Opus Postumum ${ }^{5}$, den Analoga der Geschichtsphilosophie (neben dem Schematismus der Objektbestimmung, kann man auch eine Schematismus der Analogie findet wird) bzw. den in seinen Vorlesungen und in seinem Nachlass zu findenden verschiedenen Überlegungen zum Thema der stetigen und extensiven Größen (in Beziehung mit dem transzendentale Zeitbestimmungen der Qualität und Quantität, und zwar, Zeitinhalt und Zeitreihe).

Unser Thema kann in vier grundsätzliche Achsen gegliedert werden. Zuerst wird die alte Frage nach dem Verhältnis zwischen dem Sinnlichen und dem Intelligiblen aufgegriffen. ${ }^{6}$ Zweitens kann der Sinn der Verbindung zwischen der Frage der Schematismuslehre und der Frage der Zeit und der damit verbundene Vorrang der Bedeutung von «Schema» als transzendentale Zeitbestimmung erörtert werden. Hiernach wird es interessant zu erforschen, inwieweit die Definition des Schemas als transzendentale Zeitbestimmung eine Art roten Faden für die verschiedenen Bedeutungen des Terminus «transzendentales Schema», die im Werk des Königsberger Philosophen anzutreffen sind, darstellt. Das Problem, das sich hier stellt, sollte an einigen Stellen über eine strikte Betrachtung des Schematismus in der KrV hinausgehen, und zwar in

welches als Gesetz dieses im Begriff konkretisierten Denkvorgangs und in seiner Verbindung zum «Kennen» das Subjekt zuvor mit den im Prädikat angemessenen Merkmalen in Verbindung bringt. Wenn nun das Urteil vor dem Begriff besteht, da vorherige Reflexionsprozesse zum Erkennen der diesen definierenden Merkmale erforderlich sind, stellt sich die Frage, inwieweit die Funktion des Schemas als Bedingung der Möglichkeit der Bezugnahme der Grundbegriffe auf die Erscheinungen nicht von einem anderen, grundlegenderen und an die Urteilskraft gebundenen logischen Vorgang abhängt. Und wenn dem so wäre, wie ist dann die Vorzeitigkeit der logischen Reflexionsvorgänge mit der Tatsache zu vereinbaren, dass die reflektierende Urteilskraft in KU als nicht konstitutiv vorgestellt wird?

4 Das Erhabene deutet außerdem auf eine subjektive Finalität hin, bei der aufgrund fehlender Harmonie innerhalb des Vermögens im Streben nach der Vorstellung des Nichtvorstellbaren eine Art Steigerung ins Übersinnliche stattfindet.

5 Dieser erste transzendentale Grundsatz deutet auf eine vom Raum ausgehende Rekonstruktion des Schematismus hin und ermöglicht gleichzeitig den Übergang der Grundsätze der Metaphysik der Naturwissenschaft zur Physik.

6 Daval zieht das Beispiel der verführerischen Frau als Repräsentation des Dämonischen oder sensible Darstellung des Bösen heran, als ein beispielhafter Fall der durch das Schema bewirkten Wandlung der Form, und er erinnert im selben Sinne an den Brief an Arnauld vom 30. April 1687, in dem Leibniz die metaschematismi erwähnt, und sich dabei auf die selben Verwandlungsprozesse bezieht. Der Begriff Schema zielt somit auf eine Trans-Formation, eine Wandlung der Form, ab, die die spezifische Bedeutung des Wechsels von einer intelligiblen zu einer sensiblen Form erhält. [Cfr.: Daval, R.: La Métaphysique de Kant. Perspectives sur la métaphysique de Kant d'après la théorie du schématisme, PUF, Paris, 1951, s. 6.] 
dem Sinne, in dem ein allgemeiner gefasstes Verständnis des Zeitbegriffs in der Kantschen Philosophie möglich ist, welches eine systematische Betrachtung unter Berücksichtigung der Entstehung der Lehre des Schematismus in der vorkritischen Periode sowie eine rückschauende Betrachtungsweise der Formulierung dieser Lehre in der $\mathrm{KrV}$ in ihrer Erörterung in späteren Werken erforderlich machte. In der dritten Achse geht es darum, anhand der Kategorientafel zu demonstrieren, wie jedes einzelne der Schemata in Verbindung mit den reinen Grundsätzen des Verstandes, mittels der Skandierungen Zeitreihe, Zeitinhalt, Zeitordnung und Inbegriff, das Eintreten der logischen Funktionen in die Zeit zulässt.

Die Kategorien der Quantität, die sich auf als aufeinander folgende Augenblicke auftretende anschauliche Mannigfaltigkeiten beziehen, machen die Erscheinungen, ausgehend von der transzendentalen Bestimmung der Zeit als Reihe, als extensive Größen deutlich. ${ }^{7}$ Agiert die synthesis speciosa als Reihe über eine partes extra partes erzeugte homogene Mannigfaltigkeit, haben wir es mit einer extensiven Größe zu tun, die in dem dem Grundsatz der Axiome der Anschauung gewidmeten Kapitel untersucht werden soll. In dem Maße, in dem diese Größe als quantitas in Erscheinung tritt und nach den Regeln der Einteilung der reinen Begriffe nach logischen Funktionen agiert und gleichzeitig von den gleichen reinen Formen der Anschauung regiert wird, muss die synthesis speciosa secundum quantitatem derart agieren, wie Raum und Zeit die sinnliche Pluralität der Phänomene darstellen. In dieser extensiven Reihe ist jeder Augenblick dem anderen gleich und gleichartig, unterscheidet sich aber durch sein Vorher und Nachher, und zwar in dem Maße, in dem er als Bedingung der Möglichkeit jeglicher Zählbarkeit und Nummerierung, in der die Erzeugung der Zeit selbst stattfindet, heranzuziehen ist. Beispielhaft soll nach folgendem Schema die Erörterung des Grundsatzes der Axiome der Anschauung erläutert werden, auf dessen Grundlage der extensive Charakter eines jeden Phänomens qua Größe nachgewiesen wird: Der Obersatz besagt, dass alle Phänomene eine Anschauung a priori in der Raumzeit, die ihnen als Grundlage dient, enthalten. Im Untersatz sind Raum und Zeit formelle Anschauungen, die als Juxtaposition unendlich teilbarer gleichartiger Teile definiert werden. Daraus wäre folgende Schlussfolgerung zu ziehen: Da Raum und Zeit quanta continua sind, müssen alle Phänomene der Bedingung der Möglichkeit ihrer Anschauung unterliegen, d.h. der Form der Synthesis von Raum und Zeit.

Die Erscheinungen sind jedoch auch intensive Größen, Gegenstand einer mathesis intensorum. ${ }^{8}$ Das Schema des Grades bezieht sich auf die Realität

8 Cf:: Ak, V, 173. (Prolegomena A91). 
der Dinge, also mit den Worten Wolffs: auf die determinatio positiva et vera. Die Realität ist von der Wirklichkeit der Dinge in der Welt zu unterscheiden. Dabei handelt es sich um die Art und Weise, in der die Gesamtheit der Merkmale oder Prädikate ihrem Inhesionsubjekt entsprechen. Die Bestandteile der intensiven Größen sind durch Verbindung und nicht durch Aggregation vereint, was bedeutet, dass ihre Erfassung mit einem Mal stattfindet, während bei den extensiven Größen die Teile hinzugefügt werden, bis sie eine Einheit bilden.

Im Grundsatz der Antizipationen der Wahrnehmung wird von vorherein darauf verwiesen (und daraus resultiert der Vergleich mit der $\pi \rho o ́ \lambda \eta \psi i \varsigma$ der Kanonik Epikurs), dass die Erscheinungen als intensive Größen eine gewisse Stärke oder Intensität aufweisen ${ }^{9}$. Während bei den Antizipationen eine transzendentale Fundierung der Dynamik verfolgt wird, werden in den Analogien der Erfahrung mechanische Prinzipien vorgestellt. Der allgemeine Grundsatz der Analogien besagt, dass die in den Erscheinungen der Erfahrung vorhandenen zeitlichen Bestimmungen mit den a priori-Regeln übereinstimmen müssen, wonach der innere Sinn die Repräsentationen kombiniert und die Erkenntnis möglich macht. Die Existenz der Erscheinungen in der Zeit wird von drei Regeln bestimmt: der Beharrlichkeit, der Zeitfolge und der Zugleichsein.

Das erste Gesetz dient als transzendentale Rechtfertigung des Grundsatzes der Erhaltung der Masse und bestätigt die Notwendigkeit eines jegliche Veränderungen ermöglichenden beharrlichen Substrats. Das Sukzessionsgesetz schreibt die objektive Notwendigkeit einer festen Ordnung in der Natur vor, die die Erscheinungen untereinander in Verbindung bringt, als eine Art Korrelat jener Ordnung, in der die Erscheinungen an das Bewußtsein gebunden sind. Das Gesetz der Gleichzeitigkeit entspricht dem dritten Newtonschen Gesetz der Mechanik. In der nunmehr als compositum reale verstandenen Welt befinden sich alle Phänomene in einem konstanten commercium.

Der modale Aufbau der Zeit, bzw. der Inbegriff, ist, wie im Titel Relation dargelegt, mit Synthesen der Metaphysik verbunden. Das heißt, hierbei geht es nicht um das Verhältnis zwischen den Erscheinungen, sondern um deren Verhältnis zu den Vermögen der Erkenntnis selbst. In den Postulaten des empirischen Denkens überhaupt wird auf die Möglichkeit, die Realität und die Notwendigkeit der Erscheinungen Bezug genommen. Das Mögliche wird als Korrelat von Phänomenen nebeneinander existierender Eigenschaften des aus logischer Sicht Vereinbaren angesehen. Im Vergleich zur rationalistischen Auslegung, bei der das Reale gemäß den materiellen Bedingungen der Erfahrung gebildet wird, wodurch das Mögliche durch Hinzufügen des sogenannten complementum possibilitatis zum Realen wird, fügt in der Kantschen Philosophie die Wirklichkeit nichts zur realitas hinzu bzw. liefert keinen logischen

$9 \operatorname{KrV}(\mathrm{A} 167 / \mathrm{B} 209)$. 
Inhalt eines Prädikats. Ist die Verbindung zum Realen unter den universellen Bedingungen der Erfahrung gegeben, handelt es sich um ein Schema der Notwendigkeit.

Zuletzt, versucht man zu demonstrieren, dass die transzendentale Zeitbestimmung auch eine praktische Dimension hat. In der vierten Achse soll der Nutzen des Schematismus im praktischen Bereich untersucht werden. Wie kann der Wille a priori gegenüber seinem Objekt bestimmt werden? Wie kann entschieden werden, ob eine Handlung in concreto mit dem bestimmten Fall in Verbindung steht oder unter die abstrakte Regel der Moralität fällt? Im Fall der transzendentalen Anwendung haben wir uns mit rein formalen Anschauungen auseinanderzusetzen. In der Praxis ist das Objekt der Anwendung per Definition eine bestimmte Handlung, also eine Instanz, die somit empirisch ist und der kausalen Verknüpfung unterliegt. Diese Anwendung erfolgt, zumal nicht von einer Kette logischer Funktionen ausgehend, sondern in dem Maße, in dem es sich um die Anwendung eines Gesetzes der Freiheit handelt, von einer übersinnlichen Vorstellung aus, welche jedoch auch konkret dargestellt werden möchte.

Insofern die Handlung den Naturgesetzen der Kausalität unterworfen ist, gilt ein Schematismus wie er im theoretischen Bereich beschrieben wird; das bedeutet die Mannigfaltigkeit wird nach einer logischen Regel vereint, die die Art und Weise bestimmt, in der die Erscheinungen miteinander verbunden sind. Soll jedoch das Faktum des Vorhandenseins einer vom Prinzip der darauf irreduziblen Intelligibilität beherrschten Wirkung, Möglichkeit oder Handlungsfähigkeit erklärt werden, muss das Bestehen einer anderen schematischen oder reflektierenden Vermittlung anerkannt werden. Darauf ließe sich erwidern, dass ein Verzicht auf diese Duplizität der Prinzipien noch möglich sei, was jedoch mit einem Verzicht auf die Existenz des moralischen Gesetzes einherginge. Will man versuchen, teleologisch nach den materiellen Grundlagen der Bestimmung ausgerichtete menschliche Verhaltensweisen zu erklären, wäre für die Moralität kein Platz. Diese würde sich in einem Geflecht von hypothetischen und von beabsichtigten Wirkungen oder Motivationen bedingten Berechnungen auflösen (die Erziehung bei Montaigne, die bürgerliche Verfassung bei Mandeville, das moralische Gefühl bei Hutcheson bzw. die Physik für Epikur, der Wille Gottes für Crusius oder die Vollkommenheit für Wolff oder die Stoiker). ${ }^{10}$

Die physiokratische oder streng kausale Betrachtung der Erscheinungen führt zwangsläufig zu einer ersten nicht kausalen Grundlage, wenn sie sich nicht antinomisch in einer unendlich verlängerbaren Kette zeitlicher Verbindungen zwischen Wirkungen erschöpfen möchte. Sowohl der Psychologismus

$10 C f:: \mathrm{Ak}, \mathrm{VI}, 153$ (KpV A69).

Contrastes vol. XX-Nº1 (2015) 
oder Naturalismus, welcher die Erklärung psychischer Phänomene auf die empirische Verbindung von induktiv herausgenommenen Assoziationsregeln reduziert, als auch der Dogmatismus, der die Vorstellungen ohne deren notwendige Verankerung in der Erfahrung zu kennen vermag, sowie der naive Rationalismus und der Idealismus, in denen die Beziehung zwischen Objekten und deren Vorstellungen auf eine bloße Zusammenstellung von Affektionen reduziert wird (im Idealismus ist das Ding eine Wirkung seiner Repräsentation, während im Realismus das Objekt selbst die Ursache der Repräsentation im Subjekt darstellt) erweisen sich auf theoretischer Ebene als zwecklose, dem intentionalen und konstruktiven Charakter der Erkenntnis als transzendentale Aufgabe ferne Wege. In diesem Fall bedarf es einer logischen Ebene, einer Art Geltungslogik, die zusammen mit dem faktischen Ablauf der Ereignisse in Erscheinung tritt. Auf praktischer Ebene übersteigen die die Attribuierungsverbindung zwischen dem Subjekt und seiner Handlung dominierenden Beziehungen zwischen Verdienst und Unverdienst erneut den Bereich der natürlichen Kausalität. Diese neue Ordnung lässt sich jedoch nicht über die Kausalität, sondern über die Zurechnung und den Grundsatz der Intelligibilität erklären. An dieser Stelle könnte man sich weiter fragen, ob der behauptete Gegensatz zwischen dem Prinzip der Kausalität und dem Prinzip der Zurechnung seine eigene Einteilung in heterogene Ordnungen, wie den Bereich des Seins und Sollens besitzt, oder ob dieses bereits erwähnte notwendige Einbringen dieser besonderen Form von Zeitlichkeit außerhalb der Zeit, die in der praktischen Ordnung als übersinnlich in Erscheinung getreten ist, ebenfalls im Bereich des Seins wirken kann. Mit anderen Worten: Es stellt sich die Frage, ob der Schematismus der reinen Vernunft die primäre und ursprüngliche Verständnisweise der grundlegenden Beziehung zwischen den beiden gespaltenen Welten des Intelligiblen und des Sinnlichen darstellt (der ektypische Verstand besteht eben im Kennen, Spekulieren und somit im mittelbaren und symbolischen Zugang zu den Dingen), so dass in der Praxis eine Art stellvertretender Schematismus rekonstruiert werden kann, welcher lediglich vom ersten ausgehend ableitend formuliert worden ist. Dabei ist die Bezeichnung dieser Vermittlung als Schema, wie von Kant in der Typik der reinen praktischen Urteilskraft, in der er sich fragt, ob die Verwendung des Wortes «Schema» im praktischen Bereich «schicklich ${ }^{11}{ }_{\Downarrow}$ sei, an sich schon problematisch. Oder ist es jedoch so, dass, wie sich z.B. aus den Überlegungen Kelsens zum Zurechnungsprinzip scheinbar ableiten lässt, die Festsetzung eines logischen oder intellektuellen Kerns im Bereich der reinen Vernunft nichts anderes als eine Art Verinnerlichung dieses übersinnlichen Rests oder dieser grundlegenden Notwendigkeit von Teilung, Spaltung oder Multiplikation von Grundsätzen der Intelligibilität darstellt.

11 Ak, VI, 188. KpV (A122/123).

Contrastes vol. XX-Nº1 (2015) 
Andererseits, kann man fragen, wie der Begriff Schema, der im Zusammenhang mit matematischen Problemen steht, im Wesentlichen mit dem Begriff der Konstruktion, gleichzeitig diese fundamentale Beziehung mit dem Phänomen der Zeitlichkeit haben kann.

Die Berücksichtigung der Entwicklung der Geometrie in der Zeitepoche unseres Autors sowie die Verwendung des Begriffs Schema seitens einiger Philosophen, bei der der Einfluss der Tradition von Aristoteles und des Atomismus von Epikur deutlich wird, lassen uns verstehen, warum Kant dieses eigenartige universelle Verfahren der Einbildungskraft, mit dem die reinen Begriffe des Verstands auf die Phänomene angewandt werden, mit einem Begriff wie «Schematismus« bezeichnet. Bacon verwendete z.B. den Ausdruck «schematismi ${ }^{12}$ », als er sich auf die Umwandlung der Elementarteilchen bezog. Im Rahmen seiner Lehre von der Materie, in der der Latens Schematismus eben in der Entdeckung der strukturellen Eigenschaften der Substanz begründet liegt, stellt er bereits eine Unterscheidung zwischen dem mit dem «Dichten und Sonderbaren» verbundenen Schematismus der Quantität und dem an die Vereinigung von Schwefel und Quecksilber gebundenen Schematismus der Qualität $\mathrm{an}^{13}$.

Betrachtet man den Einfluss des methodischen Paradigmas der Geometrie, wird deutlich, dass der Begriff «Konstruktion» von entscheidender Bedeutung für die Weiterentwicklung der Kantschen Schematismuslehre ist, wie in die Dissertatio bzw. in Untersuchung über die Deutlichkeit der Grundsätze der natürlichen Theologie und der Moral zu sehen ist. Darin erwähnt der Königsberger Philosoph bereits die Unterscheidung zwischen der Methode der Mathematik und der Methode der Philosophie, die er später in der transzendentalen Dialektik der KrV weiter ausführt. Die Methode der Mathematik in der Philosophie von Kant erscheint erstmals in Verbindung mit dem ungewissen Status der Metaphysik oder der damals so genannten «Hauptwissenschaft» in einer von Kant eingereichten Abhandlung für eine Ausschreibung der Königlichen Akademie der Wissenschaften Berlin im Jahre 1763. Diese Methode wird nicht nur die Art und Weise bestimmen, in der die erste Kritik im Innersten ihrer Struktur diskutiert und verstanden werden kann, sondern auch den Kurswechsel, aus dem die kritische Philosophie hervorgeht und in der die Metaphysik ihre eigene Begründungsmöglichkeit findet.

Bei den mathematischen Konstruktionen wird das Verfahren der Projektierung geometrischer Figuren von einer Regel geleitet, die mit der Exposition

12 Bacon, F. Novum Organum, Übersetzung von. C. Fernando Almori, Losada, Buenos Aires, 2003, p. 95.

13 Cfr.: Rees, G.: «Mathematics and Francis Bacon's natural philosophy» in Revue internationale de philosophie, XL, 1986, pp. (399-426). 
der Figur in einer anschaulichen Repräsentation einhergeht. Kant erläutert die Erzeugung der sicheren Erkenntnis unter Verweis auf eben diesen Konstruktionsprozess, d.h. die Legitimation der Anpassung der Begriffe an die Erscheinungen basiert auf der Konstruktion des Gegenstands selbst, und zwar als ein Konstrukt der Urteilstätigkeit des Subjekts. Analog dazu wird im transzendentalen Schematismus die Notwendigkeit deutlich, die reinen Verstandesbegriffe, welche auf einer Anschauung aufgebaut sind, darzulegen, um somit ihre Anwendung auf die Erscheinungen und die objektive Gültigkeit der Erkenntnis sicherzustellen.

Die Bedeutung der Notio der Konstruktion bei der Entstehung der Schematismuslehre wird ebenfalls in der Definition des für die Erzeugung von Schemata zuständigen Vermögens, nämlich der transzendentalen Einbildungskraft, deutlich. Die aus der Wolffschen Tradition übernommene Idee der Einbildungskraft als Fähigkeit, sich etwas Abwesendes zu vergegenwärtigen --auch wenn sie noch immer den empirischen Regeln der Assoziation unterliegt-- erfüllt eben diese konstruktive Funktion, nämlich ein Objekt dort zu erzeugen, wo es nicht vorhanden ist oder noch eine unartikulierte Myriade sinnlichen Materials darstellt.

Das Thema der Konstruktion wird im Kontext der von Kant angewandten Rechtfertigung des synthetischen Charakters a priori der Urteile der Arithmetik und der mit einigen seiner herausragendsten Kritikern angestoßenen Diskussion erörtert. Die Analyse der Hilfskonstruktionen, von denen ausgehend das synthetische Moment der mathematischen Aussagen erläutert wird, lenkt die Aufmerksamkeit auf eine der herausragendsten Formen der Erörterung der Beziehung zwischen dem Endlichen und dem Unendlichen, nämlich auf das Thema des Kontinuums, definiert als die Herausforderung, die unendliche Juxtaposition einer Reihe diskreter Elemente und gleichzeitig die Tatsache der Möglichkeit der stetigen Teilung ad infinitum einer begrenzten RaumZeit-Größe zu erklären. Dieses Thema wird wiederum von grundlegender Bedeutung für die Auslegung der mathematischen Grundsätze sein, von denen ausgehend die Schemata Zahl und Grad erörtert werden, und zwar, die zwei ersten zeitlichen Bestimmungen.

Aus dem bisher Gesagten folgt, dass das Kapitel des transzendentalen Schematismus eine vorzügliche Möglichkeit bietet, die Philosophie Kants und das Thema, das sich wie ein roter Faden durch die KrV zieht, zu verstehen. Es handelt sich dabei um die Möglichkeit der synthetischen Urteile a priori und die Eingrenzung des Begriffs des Transzendentalen, welcher wie das Schema einen temporalen Übergang zwischen den beiden unregelmäßigen Ordnungen des Sinnlichen und des Intelligiblen darstellt und gleichzeitig als dessen Terminus ad quem und Terminus a quo fungiert. Also kann das Transzendentale parallel zur Unterscheidung zwischen einer statischen und dynamischen Auslegung des 
Schematismus als das Ergebnis eines Prozesses, wo es mit dem Bereich des Transzendenten übereinstimmen würde, oder als kontinuierliche Bewegung des Vorübergehens angesehen werden. Letztere soll gemä $\beta$ einem allgemeinen dialektischen Schema des Verstehens, bei dem Identität und Unterschied gleichzeitig bestätigt werden, als ein «immanentes Transzendieren» angesehen werden. Anderseits, der Keim der Schematismuslehre erscheint untrennbar mit dem mathematischen Konstruktionskonzept verbunden. Weiterhin bliebe hier darauf hinzuweisen, dass um das Problem der transzendentale Zeitbestimmung zu verstehen, Heideggers Auslegung entscheidend ist.

Heidegger versucht, die Lehre vom Schematismus im Zusammenhang mit dem Problem der Zeitlichkeit von der Annahme her zu verstehen, dass das Programm der KrV mit dem der Rechtfertigung der Fundamentalontologie übereinstimmt. Der Meßkircher Philosoph geht von der Feststellung der Teilung des Raums des Seienden in die irreduziblen Pole des Endlichen und Unendlichen aus. Die unendliche Erkenntnis entsteht aus einer unmittelbaren Anschauung, während die endliche Erkenntnis der Vermittlung des Begriffs bedarf: es handelt sich um den korrelativen Unterchied zwischen intellectus archetypus und intellectus ectypus. Im vorangehenden Absatz ist bereits aufgezeigt worden, inwiefern sich die Metaphysik dem Konstruktionsparadigma der Mathematik anschließen sollte, wenn sie das Fundament ihrer legitimen Ansprüche zurückerlangen möchte.

Wie Heidegger in «Die Frage nach dem Ding» beschreibt, ist das mathematische Denken das vom bereits Bekannten ausgehende Lernen, das Fragen nach dem Seienden, ausgehend von den transzendentalen Bedingungen der Möglichkeit aller Erkenntnis ${ }^{14}$. Wie noch bewiesen werden soll, besteht analog dazu im praktischen Bereich ein klares Korrelat zu dieser mathematischen Vorgehensweise, und zwar dahin gehend, dass der Zweck a priori der Vernunft durch «Antizipation» gegeben ist. Diese Frage ist nunmehr von zwei Vektoren ausgehend zu erörtern. Einerseits wird in dem Maße, in dem die bereits erwähnte Spaltung des ontischen Raums zu einer Beschreibung der endlichen Erkenntnis als Ergebnis der Verbindung von zwei Vermögen, nämlich der Sinnlichkeit und der Spontaneität, führt, die Rolle einer Disziplin untersucht, deren Objekt jenes Seiende ist, das zur Erlangung einer mittelbaren und somit endlichen Erkenntnis verurteilt ist, aber gleichzeitig hinsichtlich seiner Öffnung zur Frage nach dem Sein hin ein altes Privileg für sich beansprucht, nämlich die Anthropologie.

Andererseits soll der Begriff $\pi \alpha 1 \delta \varepsilon i ́ \alpha$ bezüglich der Sichtweise Heideggers auf Platons Lehre von der Wahrheit untersucht werden. Im Bezug auf die Anthropologie kann festgestellt werden, dass im System Kants die Anthropologie

14 Heidegger, Die Frage nach der Ding (G.A. 41), p. 69 u. w. 
im rein pragmatischen Sinne erwähnt wird, obgleich es auf den ersten Blick den Anschein hatte, dass Metaphysik, Moral und Religion der Jäsche-Logik zufolge der Anthropologie zwangsläufig untergeordnet seien, also einer Logik, bei der die drei Leitfragen der kritischen Philosophie auf die grundlegendere Frage: «Was ist der Mensch?» ausgerichtet sind.

Hinsichtlich der Rezeption des Begriffs $\pi \alpha 1 \delta \varepsilon i ́ \alpha$ durch Heidegger sei angemerkt, dass im Kontext des Entstehens der Philosophie Platons den Mythen eine ethopoietische Funktion zukam, denn ihre diegetische Struktur basierte auf einzigartigen und beispielhaften Ereignissen, durch die eine Übermittlung von Werten erfolgt. Platon spricht von einem sich zwischen Mythos und Logos bewegenden glaubhaften Diskurs, der auf das Thema der Partizipation $(\mu \varepsilon \dot{\theta} \theta \varepsilon \xi 1 \zeta)$ zwischen der sinnlichen und der intelligiblen Welt verweist. Der zweideutige

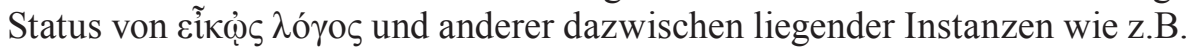

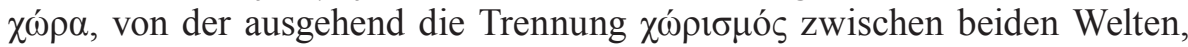
aber auch der Grund ihrer Verbindung deutlich wird, führen zu einer Bestätigung des ikonischen Charakters der Erkenntnis selbst. Diese Ikonizität lauert der gesamten Kritik weiterhin auf, die sich der Schematismuslehre zufolge der späkulären Logik nicht entziehen kann, und greift in ihrem konstitutiven Verweis auf eine vorherige Artikulierung der Kategorien, die die für sämtliche legitimen Erkenntnisse erforderliche Notwendigkeit und Universalität liefert, auf die sinnliche Mannigfaltigkeit zurück. Von diesem spekulären Schema ausgehend liegt das Grundproblem der Erkenntnis nicht in der Trennung zwischen einer intelligiblen und einer sinnlichen Ordnung begründet, deren Status stets stellvertretend zur ersten angesehen wird. In diesem Fall würde die Philosophie eo ipso zur Phänomenologie werden, und zwar zu einer Phänomenologie wie sie Lambert definierte, nämlich als eine Art transzendentale Optik von der aus man die Fehler des sinnlichen Bildes korrigieren und zum wahren geistigen Inhalt der Phänomene gelangen könnte. Das Phänomen verweist uns nunmehr auf seine Bedingungen der Möglichkeit und stellt das Ergebnis eines Prozesses des Aufbaus des Subjekts dar.

Bezüglich der Betrachtung zu $\pi \alpha \_\delta \varepsilon i ́ \alpha$ im Kontext der von Heidegger beabsichtigten Verbindung zwischen der Kritik an der Grundlegung zur Metaphysik von Kant und der Grundlegung der Ontologie ist von Bildern und wahrer Erkenntnis die Rede. In diesem Zusammenhang wird $\pi \alpha 1 \delta \varepsilon i \alpha$ als ein Übergang definiert, der beim Erkennen des Bekannten bis zu seiner perfekten adaequatio eint und trennt. Die Adäquation von Erkenntnis und Ding wird als Korrektur der Sichtweise beschrieben. Die im Wort «Bildung» enthaltene zunehmende Adäquation (ó $\mu$ oí $\omega \sigma ı)$ ) des Bildes bestimmt schließlich die Art des Übergangs von $\alpha \pi \alpha 1 \delta \varepsilon v \sigma i ́ \alpha$ zu $\pi \alpha 1 \delta \varepsilon i ́ \alpha$.

Das Problem der transzendentalen Zeitbestimmung liegt gerade in der Frage der Vermittlung begründet, also im Übergang von der Rezeptivität 
zur Spontaneität der Erkenntnis bzw. vom Universellen zum Individuellen. Die transzendentale Einbildungskraft ist also die Fähigkeit, die die Übereinstimmung von Bild und Aussehen oder Form dessen, was uns die Erkenntnis der Objekte vorausschickt, sicherstellt. Im Rahmen des Schematismus der praktischen Vernunft ist die Übereinstimmung von sinnbildlichem Werden und regulierendem Ideal bzw. von Sein und Sollen niemals konkordant oder perfekt übereinanderliegend. Das Subjekt ist im praktischen Bereich dazu verurteilt, in der Differenz, in der unmöglichen Synchronie zwischen beiden Ordnungen zu verbleiben, ist jedoch gleichzeitig dazu verurteilt, eine asymptotische Annäherung an sie zu suchen. Somit befinden sich das Sein und Sollen in einem «ursprünglichen Ungleichgewicht», wie es Duque bezeichnet, d.h. wir wissen, dass es Gott nicht gibt, müssen aber trotzdem nach seinem Gesetz handeln. Wir müssen und können dies über eine Vernunft (ratio-relatio) tun, die infolge dieser unmöglichen Übereinstimmung in die drei Ideen der Vernunft auseinanderbricht, nämlich Gott, die Welt und das tertium quid, welches seinen Übergang herbeiführen soll, d.h. der Mensch oder die Kopula, die der ursprünglichen Teilung Einhalt gebietet «Ur-teil». ${ }^{15}$

Diese Überlegung wird von Heidegger in den soeben beschriebenen Kontext der Frage der Adäquation gesetzt, wodurch er sich vom Colegium Logicum distanzierte und die Logik wieder mit der Bedeutung der Wissenschaft der Wahrheit versah. Die Frage der Wahrheit ist diesem Verständnis zufolge auch eine Frage der Anwendung der Begriffe auf die Phänomene. Dies ist wiederum das Ziel der transzendentalen Deduktion der Kategorien, was als Einstimmung auf die im Schematismuskapitel behandelte Frage der Anwendung dienen soll.

In diesem Artikel soll die Frage der Zeit im Rahmen des Schematismuskapitels untersucht werden. Somit werden die Betrachtungen der Zeit als eine Form a priori der Sinnlichkeit oder formellen Anschauung der transzendentalen Ästhetik nur in dem Maße als relevant erachtet, in dem sie zur Klärung ihres ausdrücklich in der Analytik definierten Sinnes als transzendentale Bestimmung oder Schema herangezogen werden können. In diesem Rahmen wird die Sichtweise Heideggers untersucht, wonach die Behandlung der Zeit als eine Form a priori der Sinnlichkeit, begrenzt auf die transzendentale Ästhetik, aufgrund ihrer unerbittlichen Verbindung zur Vorstellung der Sukzession als kurzsichtig erscheint. Die Charakterisierung der Zeit als Sukzession, die zu dem führt, was Heidegger und Bergson als vulgäre oder verräumlichte Konzeption der Zeit thematisiert haben, verewigt sozusagen das Schema der von Aristoteles in Physik IV aufgestellten Zeitdefinition, wonach die Kontinuität der Zeit strukturell von der Kontinuität der Bewegung abhängig gemacht

15 Cfr.: Duque, F.: «Kant: la expresión del mundo como terapia del espíritu», Contextos, III/6, 1985 s. (7-28), s. 9 u. Hölderlin, F. «Juicio y ser» in Ensayos, Hiperion, Madrid, 1983. 
wird, wobei letztere wiederum von der Kontinuität des Raumes abhängig ist. Die Betrachtung der Zeit durch Kant im Rahmen des transzendentalen Schematismus --und insbesondere hinsichtlich der Erörterung der Zahl, des ersten Schemas der Quantität, und ihrer nachfolgenden Ausführung in den Axiomen der Anschauung ausgehend vom Begriff der extensiven Größe- - hängt auf verdeckte Weise weiterhin von dieser Raumstruktur ab.

Die von der Betrachtung der Metaphysik hervorgerufene Spaltung und die Aufstellung ihrer gerechtfertigten Ansprüche führen letztlich zur Frage der Endlichkeit. Die Charakterisierung dieses endlichen Aspekts der Erkenntnis wird in einer reinen Phänomenologie der Subjektivität gelöst, aufgrund derer die schematische Abbildung der Einbildungskraft als Öffnung des Horizonts der Transzendenz dargestellt werden soll. Da der endliche Verstand der Vermittlung des Begriffs bedarf, sind wir dazu verurteilt, Phänomene kennenzulernen. Was erscheint, ist ontologisch nicht mehr dem untergeordnet, was «ist», wie es im Schema Platons der Fall ist, wo wir uns von der Erscheinung lösen müssen, um das Wesen zu finden.

Die Sichtweise Heideggers, wie sie in seiner Kritik des Psychologismus vorgebracht wird, grenzt den Bereich der Überlegung über die transzendentale Zeitbestimmung im Rahmen der Lehre des Schematismus aus den im Folgenden aufgeführten Gründen ab: Zum ersten stellt der Schematismus einen Versuch dar, das Universalienproblem der Philosophie von Locke und Berkeley vom Psychologischen (und folglich der temporalen Verkettung unterworfen) ins Logisch-Transzendentale zu verlagern. Zum zweiten sei angemerkt, dass die Rechtfertigung des synthetischen Charakters a priori der Urteile der Arithmetik, welche im Brief an Schultz vom 25. November 1788 noch präziser dargelegt ist, und die Analyse der in diesen Urteilen von den konstruktiven Prozessen der Exhibition in der Anschauung ausgehend enthaltenen Synthesis es zulassen, einen transzendentalen und nicht psychologisierenden Charakter der Rechtfertigung der Anwendung der Kategorien auf die reinen Anschauungen zu bejahen. Zum dritten, wenn der Begriff der transzendentalen Zeitbestimmung als Leitfaden der Auslegung des Schematismus wird genommen entfernt man, sich von den naturalistischen Interpretationen Kants. Zuletzt ist die metodologische Alternative, die hier ausgewählt wird, aus der Kritik an der psychologistischen Reduzierung der Wahrheit auf die Notwendigkeit und die universelle Gültigkeit der Vorstellungen zu verstehen. Dabei würde die Legalität nicht im Gedachten sondern im Denkprozess liegen. Das Verbot, Gesetze über das ideell-normative Sein der Dinge zu erlassen, ist aus empirischer Sicht an das Verständnis der Zeit als Übergang von der Logik zur Ontologie sowie an eine mögliche Auslegung des Schematismus als eine «Logik der Zeit», geknüpft.

Welche Rolle spielt die Einbildungskraft, in diesem Zusammenhang? Bereits in der Charakterisierung der wichtigen Rolle der Konstruktion in der 
Mathematik ist eine erste Annäherung an die Kant'sche Definition der Einbildungskraft unternommen worden, und zwar unter Verwendung der diesbezüglich in der deutschen Schulphilosophie angestellten Charakterisierung. Selbst wenn das Objekt nicht präsent ist, werden gemä $\beta$ den empirischen Gesetzen der Assoziation die Vorstellungen einer gleichbleibenden Regel zufolge miteinander in Verbindung gesetzt. Neben der Existenz dieser Assoziationsregeln muss sichergestellt werden, dass es eine tatsächliche Verbindung zwischen den Phänomenen gibt, die diesen empirischen Gesetzen der Ordnung der Vorstellungen entspricht. Die Grundlage a priori dieser notwendigen Verbindung zwischen den Vorstellungen bildet eine andere Art der Einbildungskraft, und zwar nicht die reproduktive, sondern die produktive. Die Einbildungskraft beschäftigt sich in ihrer transzendentalen Funktion mit der Synthesis a priori, welche wiederum die Gesetze der Assoziation, die Affinität der Phänomene und letztlich die Konstruktion der eigenen Erfahrung möglich macht. Die transzendentale Einbildungskraft verbindet das Mannigfaltige der Anschauung mit der Einheit der Apperzeption.

Der Charakterisierung der Funktion der Einbildungskraft als subiecto sub aspectum -der anschaulichen Erörterung eines Objekts- folgend ist die Differenz zwischen der Darstellung der empirischen Begriffe und der Darstellung der reinen Begriffe von besonderem Interesse. Die Darstellung der empirischen Begriffe erfolgt anhand von Beispielen, die der reinen Begriffe anhand von Schemata. Bei der symbolischen Darstellung liegt keine an den Begriff angepasste Anschauung an sich vor, weshalb das Urteilsvermögen reflektierend durch Analogie mit der entscheidenden Erkenntnis entstehen muss. Die Darstellung der Begriffe darf sich nicht auf bloße sinnbildliche und äußerliche Repräsentationen begrenzen, wie es bei den Charakterismen von Wolff oder Baumgarten der Fall ist, die eine bloße Wiedergabe der Begriffe darstellten, und die wie Zeichen in Algebra lediglich ihre Entsprechung gemä $\beta$ den Gesetzen der empirischen Assoziation zum Ausdruck bringen können und keinerlei echten Bezug zur Anschauung aufweisen. Es handelt sich hierbei um die anschauliche Darstellung des Begriffs, also die Aufgabe des Schematismus.

Mit dem Thema der Anwendung der reinen Begriffe des Verstands auf die Anschauungen (konkret: mit der transzendentalen Anwendung als Bedingung jeglicher weiterer Anwendung oder als Vorübung des Verstands bezüglich der Sinnlichkeit, bei der die Formen des Urteils bereits die Richtung des Vorgangs der figürlichen Synthese bestimmen) beschäftigt sich die transzendentale Deduktion der Kategorien. Bei der Untersuchung des Themas der Subsumtion stößt man im Zuge seiner Abhängigkeit auf die Erörterung des Begriffs «Gleichartigkeit». ${ }^{16}$ Dabei wird von der Unterscheidung zwischen verschiede-

16 Man findet eine vollständige Untersuchung über das Problem der Gleichartigkeit in 
nen Subsumtionsformen (syllogistisch und judikativ) und von verschiedenen Arten der Abgrenzung der das Subsumtionsverhältnis bildenden Begriffe an sich ausgegangen. Diese Differenzierung basiert auf dem Unterschied zwischen einem mathematischen Schematismus, einem empirischen Schematismus und einem transzendentalen Schematismus.

Das Thema des Schematismus kann ohne die Erörterung der transzendentalen Deduktion der Kategorien sicher nicht verstanden werden. In der der Königsberger Philosoph berichtet, auf welche Probleme er gestoßen ist, und zwar zunächst im Zusammenhang mit der empiristischen Tradition (mit Locke, welcher die Kategorien von der Erfahrung abzuleiten versuchte, oder mit Hume, der zwar auf den subjektiven Charakter der Projektion der Erwartung der Regelmäßigkeit in der Natur hinwies, jedoch die korrelative objektive Verbindung dieser Vorstellungen nicht erklären konnte), und später auch bezüglich der von Leibnitz angestellten abgestuften Unterscheidung zwischen cognitio clara et confusa, zumal die Trennung zwischen sinnbildlichen und intellektuellen Darstellungen eine Bedingung der Möglichkeit für die schematische Vermittlung darstellt, wie der Übergang vom Paradigma der Ähnlichkeit zum Paradigma der Gleichartigkeit beweist.

Es dürfen jedoch die jeweiligen Bereiche nicht verwechselt werden. Die zu einem späteren Zeitpunkt noch ausführlich behandelte modale Auslegung der Beziehungen zwischen Deduktion und Schematismus läuft auf eine Charakterisierung dieses Bezugs der Kategorien auf die Objekte unter dem Ternion der Kategorien Möglichkeit, Wirklichkeit bzw. Notwendigkeit hinaus. ${ }^{17}$ Dieser Hypothese folgend würde sich die transzendentale Deduktion mit der Möglichkeit und der Notwendigkeit der Anwendung der Begriffe auf die Phänomene beschäftigen, während im Schematismuskapitel die Wirklichkeit dieses Bezugs behandelt wird. Diese These ist mit unterschiedlichen Nuancen von Autoren wie Leo Freuler, Curtius oder Philonenko verteidigt worden die, obgleich sie dem Schematismus in der Linie Heideggers eine grundlegende Bedeutung beimessen, diesen als einen Gegenbeweis definieren, welcher auf psychologischer Ebene die Grundlage für die Abfassung der zweiten Ausgabe der transzendentalen Deduktion der Kategorien darstellt. ${ }^{18}$ Leo Freuler misst --unter Verweis

in: Arias Albisú, M.: «Una relación de homogeneidad entre terminus heterogéneos. El concepto de homogeneidad en el capítulo del esquematismo de la Crítica de la razón pura» en Diánoia, v. $54, n^{\circ} 63,2009$, ss. (71-88).

17 Gemäss Chipman's Ansatz, die Rechtfertigung der Anwendung des Begriffe auf die Erscheinungen wird in Rahmen des transzendentale Deduktion ausgeführt. Aber diese Verfahren mussen auch notwendig bezüglich jedes Kategorie erklären. Dieses Funktion wird für die Grundsätze gespielt. Cfr.: Chipman, L.: «Kant's Categories and their Schematism» in Kant-Studien 63, 1972, pp. (36-50).

$18 C f$ : Curtius E.R.: «Das Schematismuskapitel in der Kritik der reinen Vernunft» en: 
auf eine Fußnote, in der Kant den Verstand mit den problematischen Urteilen und die Urteilskraft mit den assertorischen Urteilen in Verbindung setzt-- der transzendentalen Deduktion eine problematische Rolle bei, welche in der Aufzeichnung der Grundlinien der Möglichkeit der Anwendung der Kategorien auf die Phänomene besteht ${ }^{19}$. Der transzendentale Schematismus zeigt jedoch das konkrete Anwendungsverfahren der einzelnen Begriffe auf.

Auch wenn beide Kapitel scheinbar eine Antwort auf das allgemeinere Problem der Anwendung der Kategorien auf die Objekte zu geben vermögen, wird das Argument geltend gemacht, wonach das transzendentale Schema nicht als ein bloßes Instrument der externen Subsumtion zur Vereinbarung der heterogenen Produkte des Verstands und der Sinnlichkeit, als voneinander gespaltene und irreduzible Vermögen, zu verstehen ist. Wir nehmen jedoch an, dass es erstens eine mit der Rezeptivität einhergehende aktive Dimension und eine der Spontaneität des Verstands innewohnende passive Dimension gibt, und dass zweitens die grundlegende Charakterisierung des transzendentalen Schemas nicht ausschließlich an den Subsumtionsprozess gebunden ist, bei dem dem Schema lediglich die Rolle des terminus medius zukommen würde.

Eine andere Flexion des Begriffs transzendentales Schema, nämlich die der Zeitbestimmung, erscheint hingegen leichter mit der Annahme vereinbar zu sein, dass der Verstand und die Sinnlichkeit Vermögen sind, die ihren Ursprung in einer «unbekannten gemeinsamen Wurzel» haben.

Was die Bedeutung der veränderten Abhandlung der transzendentalen Deduktion in der zweiten Ausgabe im Vergleich zur ersten betrifft, ist eine unvermeidliche Unterordnung der Einbildungskraft unter den Verstand ersichtlich, und zwar in dem Maße, wie die Verbindung der Phänomene untereinander von den Vereinigungsfunktionen der Kategorien abhängt. Diese Unterordnung der Synthesis der Apprehension unter die Synthesis der Apperzeption lässt sich erklären, ohne dabei die Bedeutung der Schematismuslehre in Frage zu stellen, indem von der Hypothese ausgegangen wird, dass die Einbildungskraft lediglich der auf die sinnbildliche Mannigfaltigkeit bezogene Verstand ist, bzw. der Verstand nicht aus logischer, sondern aus transzendentaler Sicht betrachtet wird.

So wie die Vermögen lediglich als ein Ergebnis ihrer Aktivität zu verstehen sind, entstehen die Kategorien als Ergebnis ihres «Angeborenseins» erst nach ihrer Anwendung auf die Erfahrung. Die Betrachtung des transzendentalen Schemas als tertium quid für die Anwendung (von den Begriffen auf die formellen Anschauungen) oder Subsumtion (von unten nach oben) führt uns zu

Kant-Studien, 19 (1-3), 1914, pp. (338-336). Philonenko, A.: «Lectura del esquematismo trascendental», Agora, 7, 1988.

19 Freuler, L.: «Schematismus und Deduktion in Kants Kritik der reinen Vernunft» in Kant-Studien, 82 (1991) pp. (397-413). 
einer ersten Annährung an der Begriff des transzendentalen Schemas, der seiner Definition der transzendentalen Zeitbestimmung vorausgesetz. Empirische Begriffe bilden sich ausgehend vom Dekantieren der allgemeinen Merkmale vieler Individuen als Repräsentationen heraus. Dabei wird induktiv durch Verallgemeinerung a particulari ad universali vorgegangen. Folglich wird davon ausgegangen, dass das, was für vieler einer gleichen Gattung angemessen ist, für alle angemessen ist. In diesem Zusammenhang kann eine gewisse Gleichartigkeit zwischen ihnen und den Objekten der Erfahrung, die unter diese subsumierbar sind, festgestellt werden. Der Unterschied zwischen dem Begriff und der Anschauung ist jedoch ein Unterschied in ihrer Natur. Es handelt sich dabei keinesfalls, wie von Empirikern und Dogmatikern interpretiert, um einen ständigen Übergang von Elementen mit unterschiedlichem Ähnlichkeitsgrad. Aus diesem Grund bedarf es im Fall der Grundbegriffe neben den Kategorien und den Anschauungen gleichzeitig eines transzendentalen Schemas als drittes gleichartiges Glied.

Was die bestimmende Definition des Schemas, die in diesem Artikel vorgestellt wird, also die der transzendentalen Zeitbestimmung betrifft, sollte auf die Ambiguität ihrer Darlegung verwiesen und versucht werden klarzustellen, ob die Zeit das Objekt, das Subjekt oder das Mittel der Bestimmung ist. Wird die Bestimmung von der Zeit vorgenommen, ist unvermeidlich davon auszugehen, dass die Anschauung das Objekt der Bestimmung darstellt. Jedoch ist die Anschauung schon per Definition formell und daher bestimmend. Im Kontext des transzendentalen Schematismus ist die Zeit als Form des inneren Sinnes eben dieses sinnbildliche Material der Anschauung. In diesem Sinne sind wir gezwungen, entweder die Existenz von zwei verschiedenen Zeiten zu akzeptieren, oder die Zeit als etwas in sich Geschlossenes anzusehen, d.h. sie ist gleichzeitig das Bestimmende, das Bestimmte und das Mittel ihrer Bestimmung. Bei Betrachtung der im Ausdruck Zeitbestimmung enthaltenen Zweideutigkeit macht sich eine Festlegung des handelnden Subjekts und des Objekts der Bestimmung erforderlich, unter Beachtung der Tatsachen, dass das, zu dem der Bestimmungsvorgang scheinbar ausgeführt wird, also die Zeit, schon per Definition durch die Einbildungskraft einen bestimmenden Charakter hat. Gleichzeitig macht die logische Funktion des Begriffs, verstanden als bestimmender Pol einer als Objekt angesehenen Zeit und nicht als Mittel zur Bestimmung, die Existenz eines Dritten oder eines formellen Mittels, das als zeitliche Skandierung die Anwendung ermöglicht, dringend erforderlich.

Diese Hypothese führt die Untersuchung folgender Fragen mit sich: Erstens: Die losen Blätter Leningrad scheinen der These des Vorrangs des inneren Sinns oder der Zeit vor dem äußeren Sinn des Raumes zu widersprechen. Das Schema ist die transzendentale Bestimmung der Zeit, und die Zeit ist gleichzeitig auch der innere Sinn. Diese Zeitdefinition setzt den Vorrang 
des inneren Sinnes vor dem äußeren Sinn voraus. Aus diesem Grund stehen wir vor dem Problem, dass eine vergleichende Betrachtung der Schlussfolgerungen der «Widerlegung des Idealismus» und der Leningrad Reflexion scheinbar zu dem Schluss führt, dass die Wahrnehmung der Phänomene in der Zeit die Möglichkeit a priori einer Veräußerlichung oder einer Verbindung zwischen dem Bewusstsein (als Sein in der Welt) und den Objekten der Welt voraussetzt. Da in unsere Vorschlag der Vorrang der Bedeutung des Schemas als transzendentale Zeitbestimmung gegenüber anderen Sichtweisen, die z.B. den Anwendungsprozess als die wichtigste Schemafunktion ansehen, bestätigt werden soll, stellen sich zwei neue Fragen. Einerseits die Notwendigkeit, zwei scheinbar unterschiedliche Arten von Zeit miteinander zu vereinbaren, nämlich die Zeit als Form a priori der Sinnlichkeit und die Zeit als Skandierung der logischen Funktionen gemä $\beta$ den vier Titel der Urteile. Ist nun der ausschlaggebende Zeitbegriff jener in der Analytik als transzendentale Bestimmung oder Schema beschriebene, und treten diese Bestimmungen aus den Begriffen hervor, muss man sich die Frage stellen, ob dies auf einen gewissen Vorrang der allgemeinen Logik über die transzendentale Logik hindeutet.

\section{AlBA JimÉnEZ RodRíGuEz (Universidad Autónoma de Madrid)}

Lineas de investigación:

Filosofía moderna, teoría del conocimiento, filosofía del derecho. Título de tesis doctoral: «Lógica del tiempo: problemas interpretativos del esquematismo trascendental kantiano y de su aplicación a la teoría jurídica de la imputación objetiva.»

\section{Últimas publicaciones:}

«Una pregunta en torno a la división de la tabla kantiana de las categorías» en Kant y las realidades en que habitamos, R. Rovira (ed.) Escolar y Mayo, 2015.

«Gleichartigkeit y Anwendung en la Crítica de la Razón Pura de Kant». Daimon. Revista Internacional de Filosofía, 2015. (En prensa)

«El concepto de complexión en Leibniz y la determinación temporal de la modalidad en la Crítica de la Razón Pura de Kant» en: Leibniz y la Ilustración alemana, Thémata (En prensa)

«El problema de la cantidad continua: Immanuel Kant y Francisco Suárez.» en Pensamiento. Revista de investigación e información filosófica. Vol. VI. Serie especial: Ciencia, Filosofía y Religión, pp. (1001-1018).

«La coacción en el marco de las lecciones kantianas sobre derecho natural» en: Violencia y abstracción en el idealismo alemán. Abada, María del Rosario Acosta y Valerio Rocco (eds.) (En prensa)

Dirección electrónica: alba.jimenez@uam.es 
